\title{
Exploring Complex Systems Aspects of Blackout Risk and Mitigation
}

\author{
David E. Newman, Benjamin A. Carreras, Vickie E. Lynch, and Ian Dobson, Fellow, IEEE
}

\begin{abstract}
Electric power transmission systems are a key infrastructure, and blackouts of these systems have major consequences for the economy and national security. Analyses of blackout data suggest that blackout size distributions have a power law form over much of their range. This result is an indication that blackouts behave as a complex dynamical system. We use a simulation of an upgrading power transmission system to investigate how these complex system dynamics impact the assessment and mitigation of blackout risk. The mitigation of failures in complex systems needs to be approached with care. The mitigation efforts can move the system to a new dynamic equilibrium while remaining near criticality and preserving the power law region. Thus, while the absolute frequency of blackouts of all sizes may be reduced, the underlying forces can still cause the relative frequency of large blackouts to small blackouts to remain the same. Moreover, in some cases, efforts to mitigate small blackouts can even increase the frequency of large blackouts. This result occurs because the large and small blackouts are not mutually independent, but are strongly coupled by the complex dynamics.
\end{abstract}

Index Terms-Blackout risk, cascading failure, complex system, electric power transmission system, infrastructure, power law, selforganized criticality.

\section{ACRONYM}

IEAR Interrupted Energy Assessment Rate

NERC North American Electrical Reliability Council

pdf probability density function

\section{NOMENCLATURE}

\author{
$a, b, c, d, A \quad$ Constants
}

Manuscript received February 13, 2010; revised March 24, 2010; accepted May 05, 2010. Date of publication February 07, 2011; date of current version March 02, 2011. This work was supported in part by the National Science Foundation under Grant ECCS-0606003, Grant ECCS-0605848, Grant SES-0623985, and Grant SES-0624361. Ian Dobson gratefully acknowledges that this paper is an account of work sponsored in part by the Power Systems Engineering Research Center (PSERC) and in part from Department of Energy grant DE-SC0002283. Ian Dobson thanks John Rundle and the Center for Computational Science and Engineering at the University of California-Davis for their generous hospitality during a sabbatical leave. Part of this research has been carried out at Oak Ridge National Laboratory, managed by UT Battelle, LLC, for the U.S. Department of Energy under contract number DE-AC05-00OR22725. Associate Editors: K. B. Misra and A. K. Verma.

D. E. Newman is with the Physics Department, University of Alaska, Fairbanks, AK 99775 USA (e-mail: ffden@uaf.edu).

B. A. Carreras is with the BACV Solutions Inc., Oak Ridge, TN 37830 USA (e-mail: bacv@comcast.net).

V. E. Lynch is with the Oak Ridge National Laboratory, Oak Ridge, TN 37831 USA (e-mail: 1ynchve@ornl.gov).

I. Dobson is with the ECE Department, University of Wisconsin, Madison, WI 53706 USA (e-mail: dobson@engr.wisc.edu).

Color versions of one or more of the figures in this paper are available online at http://ieeexplore.ieee.org.

Digital Object Identifier 10.1109/TR.2011.2104711

C Cost

DC load flow Linearized real power flow

F

$F_{i j}$

$F_{i j \max }$

$M_{i j}$

$\langle M\rangle$

OPA

Blackout frequency

Power flow through transmission line joining node $i$ to node $j$

Maximum power flow through transmission line joining node $i$ to node $j$

Fractional loading $F_{i j} / F_{i j \max }$ of line joining nodes $i$ and $j$

Fractional loading averaged over lines, a measure of network loading

Simulation of evolving network with cascading transmission line outages; Initial letters formed from the authoring institutions Oak Ridge National Laboratory, Power Systems Engineering Research Center at the University of Wisconsin, and University of Alaska.

$p_{0} \quad$ Probability for a transmission line outage due to external physical cause

$p_{1} \quad$ Probability for an overloaded transmission line to outage

$P$

$\Delta P$

\section{$\Delta P / P$ \\ $(\Delta P / P)_{c}$}

$S$

$S_{0}$

$t$

$\alpha$

$\beta$

$\lambda$
Average total load power

Power margin; Maximum total generator power minus average load power

Fractional power margin

Minimum fractional power margin; threshold for increase in generator maximum power

Blackout size

Constant

Time

Power law exponent

Power law exponent

Controls rate of increase of average load

\section{INTRODUCTION}

$\mathbf{E}$ LECTRIC power transmission systems are a key element of the national infrastructure; and blackouts of these systems have major direct, and indirect consequences for the 
economy and national security. Though large cascading blackouts in the power transmission system are relatively rare, their impact is such that understanding the risk of large blackouts is a high priority. In addition to the direct consequences of electric blackouts, the growing interconnections between different elements of the infrastructure (i.e. communications, financial markets, transportation etc.) can cause an electric blackout to impact other vital infrastructures.

While it is useful to do a detailed analysis of the specific causes of individual blackouts, it is also important to understand the complex dynamics of the power transmission network, and the frequency distribution of blackouts that it creates. There is evidence that the complex dynamics of complex systems is largely independent of the details of the individual triggers such as electrical shorts, lightning strikes, etc. In this paper, we focus on the intrinsic dynamics of blackouts, and how this complex system dynamics impacts blackout risk assessment and the mitigation of blackout risk. It is found, perhaps counter intuitively, that apparently sensible attempts to mitigate failures in complex systems can have adverse effects, and therefore must be approached with care. (Although there are a variety of definitions and types of complex systems, our main interest here is systems that behave in self-organized critical manner. Further discussion of how evolving power systems qualify as complex systems may be found in [1].)

First, as motivation for our work, we consider the statistical properties of a series of blackouts. The North American Electrical Reliability Council (NERC) has a documented list summarizing major blackouts of the North American power transmission system from 1984 to 1998 [2]. If component failures in blackouts were largely uncorrelated with each other, one might expect a probability distribution of blackout sizes to fall off exponentially (as, for example, in a Weibull distribution). However, analyses of the NERC data [3]-[6] show that the probability distribution of the blackout sizes does not decrease exponentially with the size of the blackout, but rather has a power law region. As an example, one measure of blackout size is load shed, and Fig. 1 plots on a log-log scale the empirical probability distribution of load shed in the North American blackouts. The fall-off with blackout size is approximately a power law with an exponent of about -1 .1. (An exponent of -1 would imply that doubling the blackout size only halves the probability.) Thus the NERC data suggests that large blackouts are much more likely than might be expected, which has implications for risk analysis models. Additionally, power law regions, particularly with an exponent between -1 and -2 , are consistent with those found in many complex systems models, and this helps motivate the use of such models to understand the electric power transmission system. Power law dependence of blackout probability with blackout size is also observed in Sweden [7], Norway [8], New Zealand [9], and China [10], [11]. While the NERC blackout data are the best we have found, the statistics have limited resolution because the data are limited. Moreover, there are many different mechanisms that can produce power laws [12]. Therefore the NERC data suggests rather than proves the existence of the power law region, and is consistent with complex systems models rather than conclusively validating them. Because of the potential benefits, including risk and mitigation information that

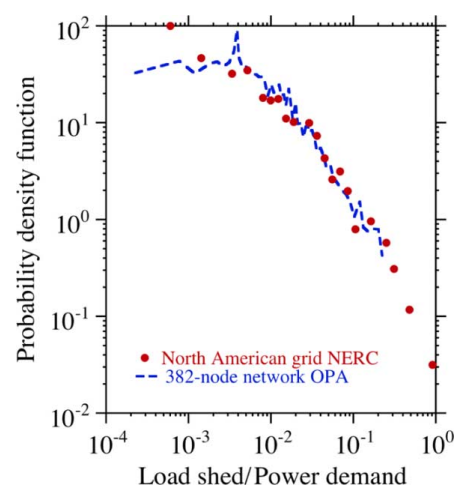

Fig. 1. Log-log plot of the scaled probability density function of energy unserved for North American blackouts 1984 to 1998 compared with the OPA model on a 382 node network.

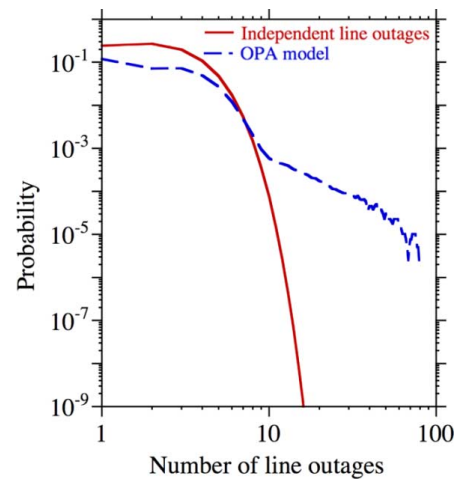

Fig. 2. Probability distribution for the number of line outages for $s$-independent line outages and cascading line outages from the OPA model on the example 382 node network.

cannot be accessed without them, modeling and simulation of the complex system dynamics is clearly indicated. Progress has been made in modeling the overall forces shaping the complex dynamics of series of blackouts. Simulations of power networks using the OPA model [13] of these complex dynamics yield probability distributions that are remarkably consistent with the NERC data, as shown in Fig. 1. OPA and the grid model are summarized in Section V.

As a simple illustration of the importance of the complex dynamics, we apply the OPA model to an idealized transmission network of 382 nodes [14], and investigate the probability distribution of blackout sizes in two different ways. First, the blackouts governed by the complex system dynamics were generated by the OPA model, and the resulting probability distribution of line outage sizes was plotted as a dashed line in Fig. 2 (the line outages shown are only the line outages that occurred when there was a blackout; that is, when load was shed). Next, the probability of any one line failing at a given time was also computed from the OPA results, and this probability was then used to construct the pdf of the blackout sizes assuming that the probabilities of outage for each line are $s$-independent of each other. This result, which is of course a binomial distribution with an exponential tail, is then compared to the OPA results in Fig. 2. The distribution of the smaller events is similar for the two calculations. However, above the size of approximately 10 line outages, the OPA model distribution diverges from the exponential, and exhibits the power law region characteristic of 
many complex systems. This is an illustration of what Charles Perrow [16] calls interactive complexity in the case of a power transmission system. The probability of, say, 20 line outages is more than 6 orders of magnitude lower using the independent probability model. This discrepancy gets even larger for a larger number of lines. The absolute probability of the large events is still very low (in good agreement with the observed probability (Fig. 1)); however, because it is many times higher than the independent probability, it plays a much larger role in the overall impact. In fact, the presence of power tails has a profound effect on risk and cost analysis for larger blackouts, particularly in the case that the power law exponent is between -1 and -2 . In this case, the large blackouts are the major contributor to the overall impact. This result bolsters the need to develop an understanding of the frequency of large blackouts and how to affect this frequency. The main purpose of this paper is to outline some of these effects, and suggest ideas to ward quantifying and mitigating the risks of larger blackouts from a complex systems perspective. This paper is an extension of the work that appeared in the conferences [17], [18]. For a survey of approaches to cascading failure analysis and simulation in electric power systems, see [19].

\section{Blackout Cost}

To evaluate the risk of a blackout, we need to know both the frequency of the blackout and its cost. It is difficult to determine blackout costs, and there are several approaches to estimate these costs including customer surveys, indirect analytic methods, and estimates for particular blackouts [20]. The estimated direct costs to electricity consumers vary by sector, and increase with both the amount of power interrupted and the duration of the blackout. Billinton [21] defines an interrupted energy assessment rate IEAR in $\$ / \mathrm{kWh}$ that is used as a factor multiplying the unserved energy to estimate the blackout cost. That is, for a blackout with size measured by unserved energy, the direct cost in dollars is

$$
C=\mathrm{IEAR} \times \text { Unserved energy. }
$$

There are substantial nonlinearities and dependencies not accounted for in (1) [20], [22], [23], but expressing the direct costs as a multiple of unserved energy is a commonly used crude approximation. However, studies of individual large blackouts suggest that the indirect costs of large blackouts, such as those resulting from social disorder, are much higher than the direct costs [20], [24]. Also, the increasing and complicated dependencies of other infrastructures mentioned earlier on electrical energy tend to increase the costs of blackouts [25], [26]. Setting aside these considerations, we will use (1) as one way to calculate the direct cost of blackouts. In particular, we will use the NERC data for the duration and power lost in North American blackouts [2] to compute the unserved energy as the product of the power loss times the duration of the blackout to obtain the direct cost

$$
C=\mathrm{IEAR} \times \text { Power loss } \times \text { Duration. }
$$

Equation (2) is a crude approximation given the uncertainties in the data; but for a statistical evaluation, we hope to capture some of the systematic trends in the dependence of the cost on blackout size.

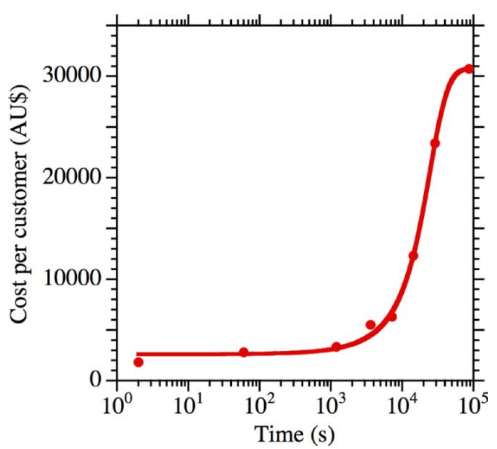

Fig. 3. Example of a fit using (3) to the data on blackout cost of the industrial customers of Australia, as given in [27]. The fit has parameters $a=13925$, $b=1.2016, c=16200$ seconds, and $d=19950$ seconds.

An alternative way of evaluating blackout costs uses customer surveys. In the report [27], there are data from several countries on the cost of power interruptions per customer. These costs are separated for residential, commercial, industrial, and large users. In several countries, the data cover blackout durations from seconds to 24 hours. The best documented data in [27] come from Great Britain and Australia.In those countries, the cost of interruptions as a function of the duration of the blackout fits very well with the following function.

$$
\text { Cost per customer }=a\left[1+b \tanh \left(\frac{\text { Duration }-c}{d}\right)\right],
$$

where $a, b, c$, and $d$ are free parameters, which we have determined by fitting the British and Australian data, as illustrated in Fig. 3. Because the same functional form seems to apply to residential, commercial, industrial, and large users, we use the functional form (3) as a representative of the cost of interruption for all customers, and use the parameter values $b=2.07$, $c=7435$ seconds, and $d=34999$ seconds that are averaged over the different types of customers ( $a$ does not matter for computing cost and risk measured in arbitrary units). We then multiply this averaged cost by the total number of customers. This multiplication leads to the following form for the direct cost as a function of the number of customers and blackout duration.

$$
C=a\left[1+b \tanh \left(\frac{\text { Duration }-c}{d}\right)\right] \times \text { Customers }
$$

In particular, to compute the direct cost, we will use (4) and the NERC data for the duration and number of customers disconnected in North American blackouts. One problem with the data and (4) is that they only apply to blackouts in duration up to 24 hours, and here we are interested in large blackouts that can last more than 24 hours. We can just add the hyperbolic tangent part of the cost for any hours past the initial 24 hours, or do a linear extrapolation. We have done this extrapolation both ways, and we have not found much difference in the scaling of the cost with the size of the blackout.

\section{Blackout Risk ANAlysis AND POWER TAILS}

The previous section approximately quantified the blackout direct cost $C$ in two ways, based on unserved energy, or on the number of customers disconnected and blackout duration. This section uses the NERC data to estimate blackout risk and its 


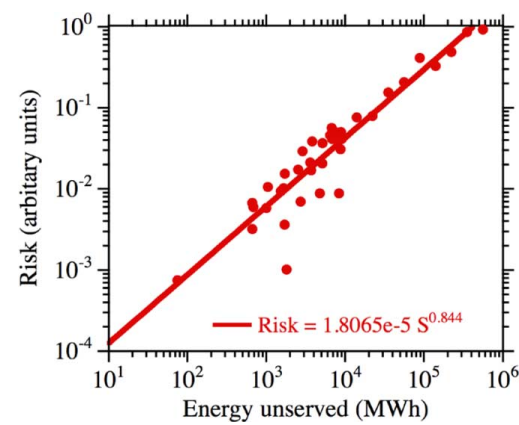

Fig. 4. Risk of a blackout as a function of the size of the blackout measured by the energy unserved, and using (2) to obtain the blackout cost.

dependence on blackout size $S$ measured either by unserved energy, or by the number of customers disconnected. Then we discuss the power laws in the frequency of blackouts and their impact on the risk.

The NERC data give the power lost, duration, and number of customers disconnected for each blackout. ${ }^{1}$ We compute for each blackout the cost using either (2) or (4), and the energy unserved as the product of power lost and duration. We also can use the NERC data to estimate the blackout frequency $F$ as a function of blackout size $S$ measured either by unserved energy, or by the number of customers disconnected. The risk of each blackout is then the product of blackout frequency and $\operatorname{cost}^{2}$ :

$$
\text { Risk }=F \times C .
$$

Equation (5) estimates the risk up to an arbitrary multiplicative constant because we have not accounted for the absolute value of the cost. Fig. 4 plots risk as a function of blackout size $S$ measured by energy unserved, and with blackout cost (2). The risk is well approximated by a power law

$$
\text { Risk } \sim S^{\beta}
$$

where $\beta=0.84$. Figs. 5 and 6 plot the risk as a function of the blackout size $S$ measured using number of customers disconnected, and with blackout cost (2), and (4) respectively. The risk is approximated by the power law (6), but the exponent depends on the way costs are calculated, with $\beta=0.29$ for the cost (2), and $\beta=0.95$ for the cost (4). All these results give a substantial increase in risk as blackout as size increases, which means that the direct cost of blackouts is dominated by the largest sizes. If we also account for the indirect costs of large blackouts, we expect an even stronger weighting of the cost for larger blackouts relative to smaller blackouts. From this result, we conclude that, although large blackouts are much rarer than small blackouts, the risk of large blackouts is significantly greater than the risk of small blackouts.

\footnotetext{
${ }^{1}$ There are missing entries for some blackouts that require these blackouts to be omitted from the calculations. We are not aware of any pattern in the omitted data that could potentially bias the results.

${ }^{2}$ In detail, the blackout frequency for size $S$ is proportional to the number of blackouts in a bin of blackout size $[S, S+\Delta S]$. In the case of blackout size $S$ measured by the number of customers disconnected, there are several different blackout costs in $[S, S+\Delta S]$ due to the dependence of (4) on blackout duration, and we average over these to compute the average risk of the blackouts in $[S, S+\Delta S]$.
}

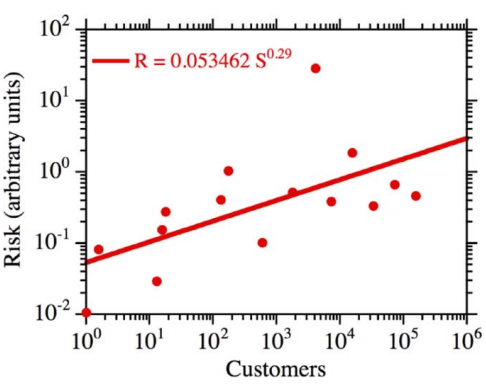

Fig. 5. Risk of a blackout as a function of the size of the blackout measured by the number of customers, and using (2) to obtain the blackout cost.

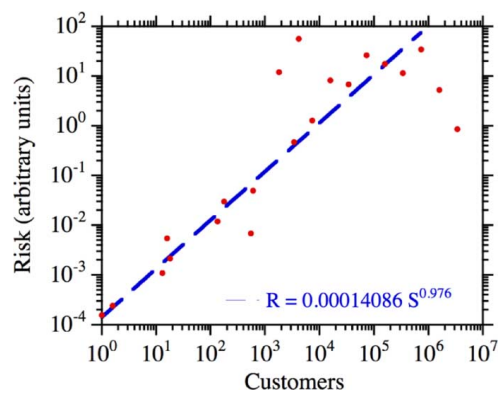

Fig. 6. Risk of a blackout as a function of the size of the blackout measured by the number of customers that lost power, and using (4) to obtain the blackout cost.

The NERC data indicates that the blackout probability as a function of the size $S$ measured by the number of customers disconnected is very well described by a function of the form

$$
F(S)=\frac{\alpha-1}{S_{0}} \frac{1}{\left(1+\frac{S}{S_{0}}\right)^{\alpha}}
$$

with the exponent $\alpha$ very close to 2. (Using other measures for the blackout size, one gets the same functional form, but some variation in the value of $\alpha$.) Therefore, the frequency of the blackout for large blackouts can be approximated as a power law

$$
F(S) \sim S^{-\alpha}
$$

The power law (8) combined with the blackout cost (2) or (4) yields the power law in the blackout risk as a function of blackout size $S$, and the greater risk of the large blackouts.

In contrast, consider the same risk calculation if the blackout frequency decreases exponentially with size so that

$$
F(S)=A^{-S} \text {. }
$$

With the simple accounting for direct costs only, we get

$$
\text { Risk } \sim S A^{-S},
$$

for which the risk peaks for blackouts of some intermediate size, and decreases exponentially for larger blackouts. Then, unless one deals with an unusual case in which the peak risk occurs for blackouts comparable to the network size, we expect the risk of larger blackouts to be much smaller than the peak risk. This is likely to remain true even if the indirect blackout costs are accounted for unless they are very strongly weighted (exponentially for example) toward the large sizes. 
While there is some uncertainty in assessing blackout costs, and especially the costs of large blackouts, the analysis above suggests that, when all the costs are considered, power law regions in the blackout size frequency distribution will cause the risk of large blackouts to exceed the risk of the more frequent small blackouts. This is strong motivation for investigating the complex dynamics of series of blackouts that can lead to power law regions.

If one is able to develop a model for the probability distribution function based on the complex systems dynamics, by normalizing the pdf to the observed frequency of the more common small blackouts, we could construct the frequency distribution. This would allow the evaluation of realistic frequencies of the occurrence of rare large blackout events that are so important in risk analysis. Additionally, by comparing the width and shape of the small blackout region of the pdf, one might be able to determine how close to the critical point the system is.

We now put the issue of power law regions in context by discussing other aspects of blackout frequency that impact risk. The power law region is of course limited in extent in a practical power system by a finite cutoff near system size corresponding to the largest possible blackout. More importantly, the risk is impacted by the frequency of smaller blackouts, and hence the shape of the frequency distribution away from the power law region. Also significant is the absolute frequency of blackouts. When we consider the effect of mitigation on blackout risk, we need to consider changes in both the absolute frequency, and the shape of the blackout frequency distribution.

\section{Mitigating FAilures In COMPLEX Systems}

Large disruptions, or normal accidents in Perrow's terminology [16], can be intrinsic to large infrastructures such as power transmission systems. Measures to mitigate these risks are not always easy to determine, and some of the mitigation measures may backfire, making the problem worse [28]. This phenomenon is also well-known in the complex dynamics of systems displaying self-organized criticality [29]-[32]. A selforganized critical system is one in which the nonlinear dynamics in the presence of perturbations organizes the overall average system state near to a critical state that is marginal to large disruptions. These systems are characterized by a spectrum of spatial and temporal scales of the disruption that exist in remarkably similar forms in a wide variety of different physical systems. Systems that operate near criticality have power laws; the frequency of large disruptions decreases as a power function of the disruption size. This is in contrast to Gaussian systems or failures following a Weibull distribution in which the frequency decays exponentially with disruption size.

The success of mitigation efforts in self-organized critical systems is strongly influenced by the dynamics of the system. One can understand self-organized critical dynamics as including opposing forces that drive the system to a dynamic equilibrium near criticality in which disruptions of all sizes occur (see [6], [13], [33] for an explanation in the context of power systems). Power laws are a characteristic feature of this dynamic equilibrium. Unless the mitigation efforts alter the self-organization forces driving the system, the system will be pushed to criticality. To alter those forces with mitigation efforts may be quite difficult because the forces are an intrinsic

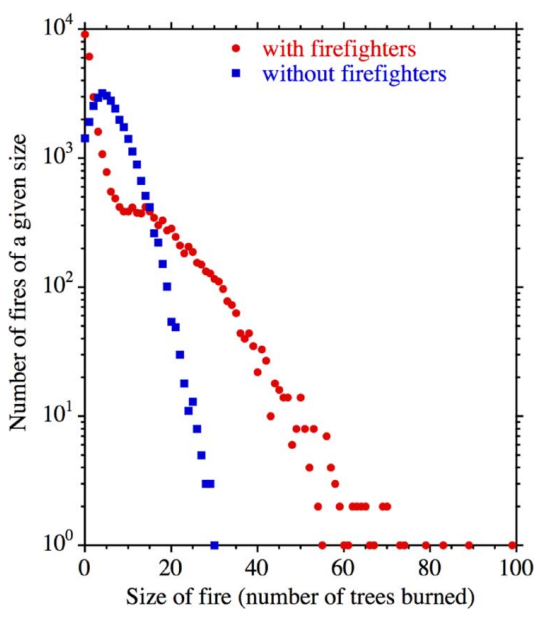

Fig. 7. Numbers of forest fires of varying size with and without fire fighting.

part of our society. Then the mitigation efforts can move the system to a new dynamic equilibrium while remaining near criticality and preserving the power law. Thus, while the absolute frequency of disruptions of all sizes may be reduced, the underlying forces can still cause the relative frequency of large disruptions to small disruptions to remain the same.

Moreover, in some cases, efforts to mitigate small disruptions can even increase the frequency of large disruptions. This occurs because the large and small disruptions are not independent, but are strongly coupled by the dynamics. Before discussing this result in the more complicated case of power systems, we will illustrate this complex systems phenomenon in the context of a forest fire model [30]. The objective of this illustration is only to explain a complex systems effect known in another application with an easier model description than power systems. While it is not surprising that the corresponding power systems results in Section VI are analogous, the validity of the power systems results needs, of course, to be considered independently from any analogy.

The forest fire model has trees that grow with a certain probability, lightning which strikes (and therefore lights fires) with a certain probability, and fires that spread to neighboring trees (if there are any) also with a given probability. The opposing forces in the forest are tree growth and fires, which act to increase and decrease the density of trees respectively. The forest settles to a dynamic equilibrium with a characteristic average density of trees. The rich dynamics of this model system has been extensively studied [30]. In our version of the forest fire model, there are two types of forests. The first type is an uncontrolled forest in which the fires are allowed to burn themselves out naturally. The second type of forest has an efficient fire-fighting brigade that can extinguish small fires with a high probability. At first, this support appears to be good; after all, we want to decrease damaging fires. However, in the longer run, the effect of the fire fighting is to increase the density of flammable material (trees). Therefore, when one fire is missed, or a few start at once (from multiple lightning strikes), the fire brigade is overwhelmed, and a major conflagration results. (This seems to be the cause of the large fires in the southeastern United States in 2001.) The enhanced probability of large fires can be seen in Fig. 7 in which the frequency distribution of fire sizes is plotted 
for the two different situations. In the case where the small fires are efficiently extinguished, the large fire tail of the distribution is significantly increased over the case with no mitigation. This type of behavior is typical because, in a complex system, there is a strong nonlinear coupling between the effect of mitigation and the frequency of the occurrence. Therefore, even when mitigation is effective and eliminates the class of disruptions that it was designed for, it can have unexpected effects such as an increase in the frequency of other disruptions. As a result, the overall risk may be worse than the case with no mitigation.

\section{SUMMARY OF OPA}

To study the real impact on the system of different mitigation measures, we use the OPA model of blackout dynamics. This section summarizes OPA, and the grid models used. OPA and the artificial grid model are described in detail in [13]. Another perspective on the complex dynamics of OPA is given in [1], and OPA is placed in the context of other cascading failure models in [33].

In the OPA model, the dynamics of blackouts involve two intrinsic time scales. There is a slow time scale on the order of days to years, over which load power demand slowly increases, and the network is upgraded in response to the increased demand. The average load power demand slowly increases at a fixed rate by multiplying the average load by a fixed number slightly greater than one at the start of every simulated cascade. There are two sorts of upgrades. Transmission lines are upgraded as engineering responses to blackouts and maximum generator power is increased in response to the increasing demand. The transmission lines selected for upgrade are those transmission lines involved in a blackout. The transmission lines are upgraded by increasing their maximum flow limits. The generation upgrade is done as needed to maintain coordination with the transmission line upgrades. In particular, the generation is increased at randomly selected generators subject to coordination with the limits of nearby lines when the generator capacity margin falls below a threshold [6]. These slow opposing forces of load increase and network upgrade self organize the system to a dynamic equilibrium. As discussed elsewhere [13], this dynamical equilibrium is close to the critical points of the system [14], [33], [34].

In the OPA model, there is also a fast time scale, of the order of minutes to hours, over which cascading transmission line overloads or outages may lead to blackout. Cascading blackouts are modeled by overloads and outages of lines determined in the context of a standard DC load flow model of the network power flows and generator power dispatch optimized by linear programming. Each simulated cascade starts from a solution of a standard DC load flow. The initial disturbance is modeled by $s$-independent outage of each transmission line with a given probability $p_{0}$. If any lines are in a state of outage, the power flows and injections are recomputed using the DC power flow equations and standard linear programming optimization of the generation redispatch [35]. The linear programming of the generation redispatch can shed load, but a large weighting factor in the cost function ensures that load shedding is avoided where possible. The redispatch of generation and load shedding are the only corrective actions modeled during the cascade, and other operator actions or remedial action schemes are not modeled.
A cascading overload may start if one or more lines are overloaded during the solution of the linear programming problem. These overloaded lines are assumed to be the lines that are vulnerable to further outage, and this is implemented by assuming that there is a probability, $p_{1}$, that an overloaded line will suffer an outage. When a solution is found, the overloaded lines of the solution are tested for possible outages. If an outage is found, a new solution is calculated. This process can lead to multiple iterations, and this cascading process continues until a solution is found with no more line outages. Then the total load shed and lines tripped are recorded. The cascade is considered to cause a blackout if a non-trivial amount of load is shed. The overall effect of the process is to generate a possible cascade of line outages that is consistent with the network constraints and the linear programming optimization. OPA represents in a simplified way the physics and engineering of important mechanisms of cascading blackouts, namely transmission line overloads and outages and the evolution of the network in response to blackouts and load demand. However, blackouts are extremely complicated, and there are dozens of physical, socio-economic and human mechanisms that combine together in blackouts. The state of the art in analyzing and simulating blackouts typically represents only a small selection of the observed mechanisms [19]. Although the OPA model includes very simple representations of the parts of the power transmission system, it can nevertheless as a combined model yield complicated complex system behaviors.

In this paper, OPA is run on artificial power grid networks, or the standard IEEE test network of 118 nodes [15]. The artificial networks are constructed by first generating a tree network with three transmission lines incident on each node, and then adding transmission lines to the circumference to join pairwise all the nodes at the largest tree depth [14]. Although this network is artificial, it has an advantage of allowing similar networks of different sizes to be generated simply by varying the tree depth in the first stage of the network generation. In this paper, we use the above method to generate artificial networks of sizes 46 , 190, and 382 nodes.

\section{Assessment of Mitigation Measures}

The OPA model allows us to study the dynamics of blackouts in a power transmission system. This model shows dynamical behaviors characteristic of complex systems, and has a variety of transition points as power demand is increased [14]. In particular, we can assess some generic measures that may be taken for blackout mitigation, and the OPA model provides some guidance on when and how such mitigation methods may be effective.

To experiment with possible mitigation effects, we consider three types of mitigation measures.

1 Require a certain minimum number of transmission lines to overload before any line outages can occur. This suppression of outages with only a few lines overloaded could represent operator actions that can effectively resolve overloads in a few lines, but are less effective for overloads in many lines.

2 Reduce the probability that an overloaded line outages. This mitigation strengthens the transmission lines. For example, it could roughly represent the effect of increased emergency ratings so that an overloaded line would be 


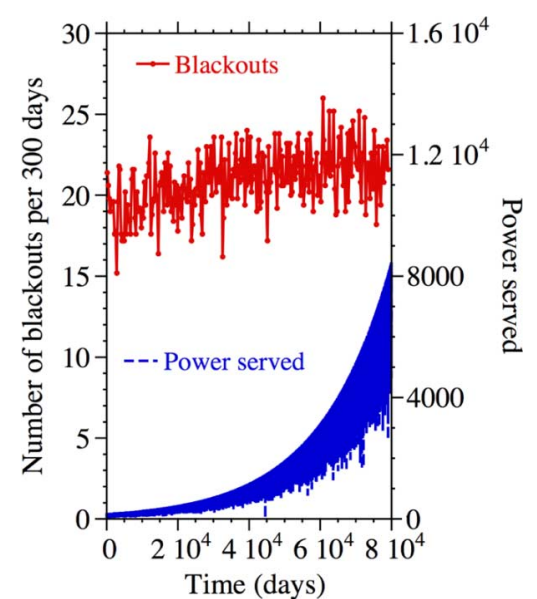

Fig. 8. Time evolution of the power served and number of blackouts from the OPA model on 382 node network.

more likely to operate while the operators resolve the line overload.

3 Increase the generation margin. This implies having greater power reserves available in the network to respond more effectively to fluctuations in the power demand. Clearly an increase in generator power available should reduce the chances of blackouts.

In what follows, we discuss each of these three options from the perspective of the OPA model. The strong dynamical correlations observed in the results of the model will manifest in several unpredicted consequences of these mitigation techniques. In these studies, we have used the 382 node network [14] and the IEEE 118 node network [15]. We note our assumption that the mitigation methods work correctly in the sense of working as designed. Although mitigation methods may sometimes fail in practice, this is a reasonable starting assumption.

We collect the data for our statistical studies during the steady state regime in the dynamical calculation. Here, steady state is defined with relation to the dynamics of the blackouts, because the power demand is constantly increasing, as shown in Fig. 8. The time evolution in the OPA model shows two distinct stages. At the beginning, and depending on the details of the initial conditions, there is a transient period. This period is followed by steady state evolution with stationary blackout statistics. This relationship is illustrated in Fig. 8, where we have plotted the number of blackouts in 300 days as a function of time. We can see a slight increase in the average number of blackouts during the first 40000 days. This transient period is followed by a steady state where the number of blackouts in an averaged sense is constant. Note that the properties in the slow transient are not very different from the steady state. However, for statistical analysis, it is better to use the steady state information. The length of this transient depends on the rate of growth in power demand. In the calculations presented here, this rate has been fixed to $1.8 \%$ per year. In the following calculations, we evaluate the statistics on blackouts by neglecting the initial transients, and doing the calculations for a time period of 80000 days in steady state. It is arguable whether the real electric power grid reaches steady state. However, it is pragmatic to begin the study of the complex system dynamics by examining their effect on the long-term steady state.

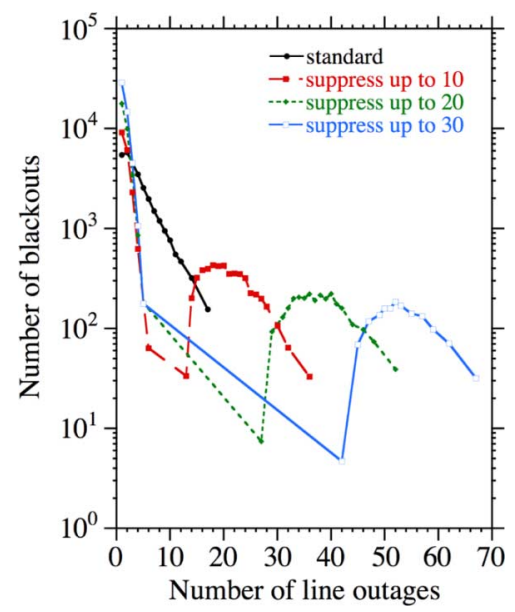

Fig. 9. Logarithm of the number of blackouts as a function of the number of line outages for different suppressions of smaller outages on the IEEE 118 node network.

\section{A. Suppressing Line Outages When Only a Few Lines Overload}

There are two possible sources of line outages in the OPA model. One is a random event causing a physical outage (for instance, a tree shorting out a transmission line). Such events happen with a prescribed probability $p_{0}$. The first type of line outage is not affected by the mitigation measure. The second cause of line outages is line overloading during a cascading event. We assume that there is a probability $p_{1}$ for an overloaded line to outage. Here we assume that operator actions can effectively resolve overloads in a few lines; therefore we require a minimum number of transmission lines to overload before allowing those lines to outage.

We implement this mitigation measure in the OPA model by suppressing line outages unless there are more than a certain number of overloaded lines. The expected result of this mitigation measure is the reduction of the blackouts involving a small number of line outages. We have used the IEEE 118 node network (178 transmission lines) for these calculations [15]. In the calculations presented here, the maximum individual load demand fluctuation is $60 \%$, and the minimum generation margin is $30 \%$. When we consider the base case (no mitigation measure applied), we found that only $9.7 \%$ of the blackouts had more than 10 line outages, and only $4.7 \%$ of the blackouts had no line outages. Therefore, the bulk of the blackouts, $85.6 \%$, had 1 to 10 line outages. This result suggests that implementing the measure that there are no line outages unless, for instance, there are at least 10 overloaded lines, should reduce substantially the total number of blackouts. As we discuss below, that is not the case.

Fig. 9 plots the logarithm of the number of blackouts as a function of the number of line outages. The logarithmic vertical scale emphasizes the rarer large blackouts, but this is appropriate given the risk analysis presented above. We can see that, with no mitigation, there are blackouts with line outages ranging from zero to 20 . When we suppress outages unless there are a certain number of overloaded lines, there is a clear increase in the number of blackouts with line outages greater than that number of lines. There is also an increase in the number of blackouts with no line outage. In particular, for suppressing outages with up to 10 lines, blackouts with 1 to 10 outages are reduced by 


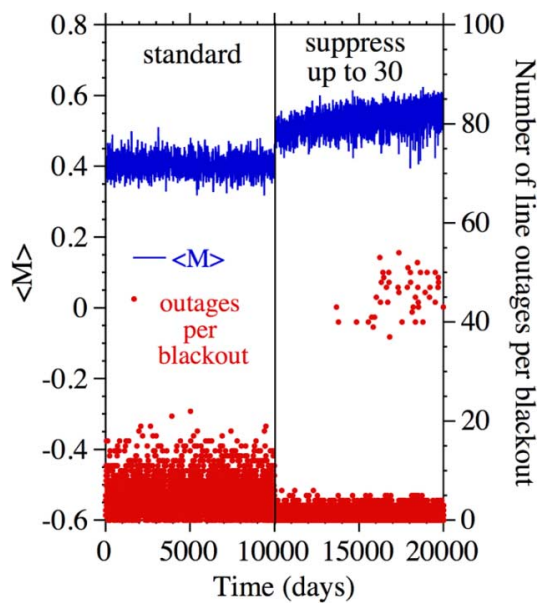

Fig. 10. Time evolution of the number of outages per blackout, and the network loading $\langle M\rangle$ before and after the mitigation of suppressing outages with less than 30 lines goes into effect on the 382 node network.

$40 \%$. However, blackouts with no line outage, or with more than 10 line outages, increase by $110 \%$. The overall result is only a reduction of $15 \%$ of the total number of blackouts. Furthermore, as the number of large blackouts has increased, this reduction on blackouts may not lead to any overall benefit to the consumers.

As there is a significant increase of rare but very large blackouts, this mitigation may have an overall negative economic effect. If we measure the economic impact of a blackout as being proportional to the power loss, we can make an estimate of the blackout cost reduction due to the reduction in the frequency of outages. If the number of transmission lines for which outages are suppressed is kept below 10, then there is a decrease in the cost of the blackouts. However, if the number of transmission lines for which outages are suppressed is larger than 10, then there is an increase in the blackout cost. However, the increase in the blackout cost is at most $60 \%$.

It is interesting to explore in more detail the consequences of implementing such a measure. To do so, we can look in detail at the time evolution of the system after the measure has been applied. In Fig. 10, we plot the number of outaged lines during a blackout as a function of time. We start with the base case, and can see that during this initial phase the number of outages per blackout oscillates between 0 and 20 , as expected from the pdf in Fig. 9. At time $t=10000$, the mitigation goes into effect, and we require 30 transmission lines to overload before any overloaded line outages can occur. Fig. 10 shows that the number of line outages per blackout is immediately reduced. The frequency of blackouts is also immediately reduced as the mitigation goes into effect, but the frequency start to slowly increase to its steady state value. At first, after the mitigation goes in to effect, there are very few blackouts with a high number of line outages, but their frequency increases until it reaches the expected value from the steady state calculation. It is because of the dynamics induced by the growth of the demand that the system self-organizes to a new dynamical state in which the improvements introduced by the mitigation are eroded, and an overall situation worse than that with no mitigation emerges.

In Fig. 10, we have also plotted a measure $\langle M\rangle$ of the network loading. $M_{i j}=F_{i j} / F_{i j \max }$ is the fraction of loading of the line connecting nodes $i$ and $j$. (Here $F_{i j}$ is the power flow

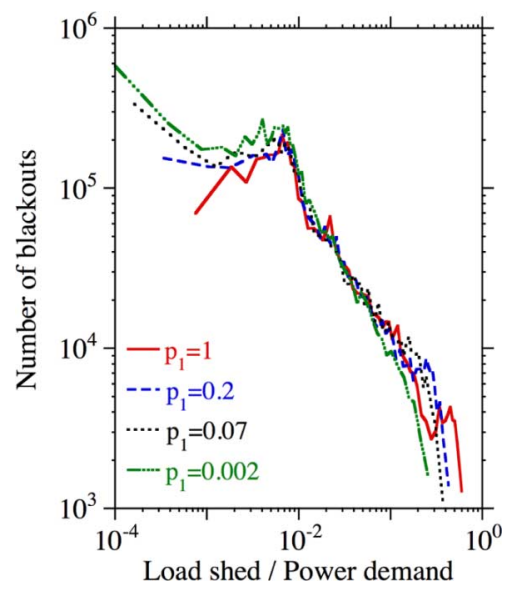

Fig. 11. Frequency distribution of blackouts for different values of the probability for an overloaded line to outage on a 190 node network.

through this line, and $F_{i j \max }$ is the power flow limit.) The averaged value of $M_{i j}$ over all the lines of the system, $\langle M\rangle$, gives a measure of how close to its transmission limits the system is operated. In the example plotted in Fig. 10, we can see a jump in the value of $\langle M\rangle$ as soon as the mitigation measure is implemented. This jump is followed by a slow evolution to ward a higher fraction of overloading. As the operators have learned to deal with up to 30 overloaded lines without line outages, the system has been operated with more lines closer to their limits. When an incident happens that triggered a blackout, this higher level of overloading makes a large blackout more likely.

Because of the time taken to reach to the new steady state with large blackouts, it is clear that in a real system it will be difficult to determine that the mitigation introduced at time $t=10000$ is the cause of the situation at a time $t=15000$ more than 13 years later. The transition time is a function of how drastic the mitigation is. For instance, for suppressing outages with up to 10 lines, the transition time to a larger blackout is barely detectable, although the largest blackouts also appear a few years later.

\section{B. Reducing the Probability That an Overloaded Line Outages}

Strengthening the transmission lines can be represented by a reduction in the probability that an overloaded line outages. The expectation from this mitigation method is an overall decrease in the frequency of the blackouts. Furthermore, multiple blackouts are also expected to be less likely because of the decreased probability of failure of each of the components. In Fig. 11, we show a plot of the distribution of the number of blackouts as a function of the load shed for different values of the probability of line outage, $p_{1}$.

As expected, we see that reducing $p_{1}$ reduces the probability of large blackouts. However, this is not the only change observed in the dynamics. With the decrease of large blackouts, there is a concomitant increase in the number of small blackouts. The overall result is that there is hardly any change on the frequency of blackouts. In the intermediate range, where the probability distribution of the load shed varies inversely proportional to the size of the blackout, there is not much change. The functional form remains algebraic with exponents close to 1 . This robustness of the algebraic tail of the pdf is characteristic of self-organized critical system [31]. 


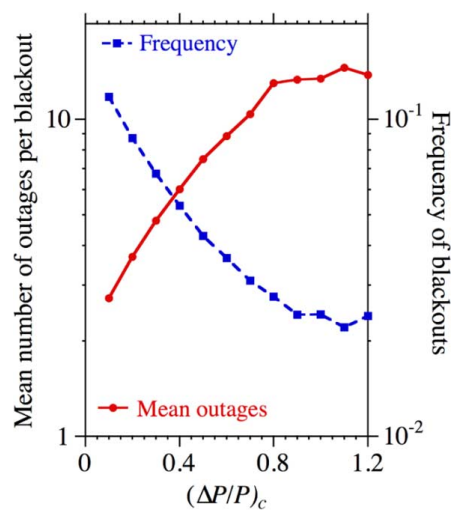

Fig. 12. Frequency of blackouts, and mean number of line outages per blackout as a function of $(\Delta P / P)_{c}$ on the 46 node network.

Because the OPA model represents the power system in a simplified form that lumps together multiple effects, the mitigation measures that we can represent with OPA are limited, and may be implemented in several ways. For example, there may be several ways to upgrade the power system to reduce the overall probability $p_{1}$ that an overloaded line outages, such as increasing line ratings or operational rules that provide more margin. Although the initial parts of cascading sequences can be understood, it remains a challenge to assess the average effect of upgrades on the subsequent parts of long cascading sequences, even when complex systems effects are neglected.

\section{Increasing the Generation Margin}

In power systems, the maximum total generation power available exceeds the average load $P$ by an amount $\Delta P$. The generation margin can then be expressed as the fraction $\Delta P / P$. In the OPA model the maximum generator power is increased when the generation margin falls below the minimum generator power margin $(\Delta P / P)_{c}[13]$.

Increasing $(\Delta P / P)_{c}$ is possibly the simplest mitigation approach [36], and we expect a reduction of the overall number of blackouts. We find that the frequency of blackouts decreases as the capacity margin increases. We have carried out these calculations for several idealized networks. In Fig. 12, we have plotted the frequency of blackouts as a function of $(\Delta P / P)_{c}$ for an idealized network with 46 nodes. The frequency decrease with increasing $(\Delta P / P)_{c}$ only happens when this margin is greater than the standard deviation of the load demand fluctuations. When they are comparable, there are no simple mitigation measures that are effective in reducing the blackout frequency. Also note the increase in mean blackout size (measured by the number of line outages) as blackout frequency decreases in Fig. 12. When we increase the generator margin, the character of the blackouts changes. When the generator margin is small, the blackouts are of small size with mostly no line outages. However, at high generation margin, they became considerably less frequent, but have a large size with many line outages. This pattern is illustrated in Fig. 13, where we have plotted the number of blackouts for a given number of line outages for different values of $(\Delta P / P)_{c}$. This result suggests that the increases on generator margin need to be associated with upgrades of the transmission grid.

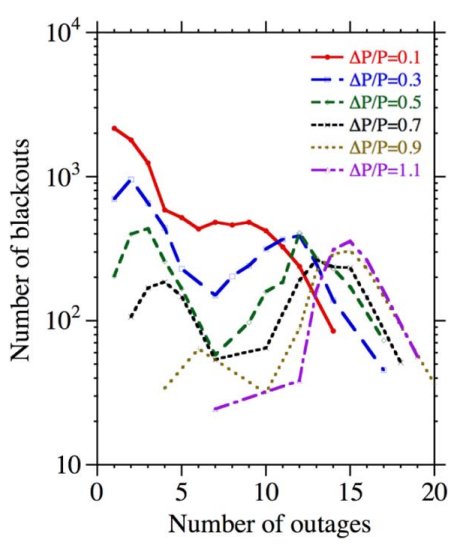

Fig. 13. Frequency of blackouts as a function of number of line outages for different values of $(\Delta P / P)_{c}$ on the 46 node network.

\section{CONCLUSIONS}

Complex system dynamics in the power transmission system has important implications for mitigation efforts to reduce the risk of blackouts. As expected from studies of general self-organized critical systems, the OPA model shows that apparently sensible efforts to reduce the risk of smaller blackouts can sometimes increase the risk of large blackouts. This result is due to the nonlinear interdependence of blackouts of different sizes caused by the dynamics. The possibility of an overall adverse effect on risk from apparently sensible mitigation efforts shows the importance of accounting for complex system dynamics when devising mitigation schemes.

When we apply mitigation measures that tend to reduce the probability of small blackouts, we can often see an increase in the frequency and/or the size of large blackouts. Conversely, when we try to eliminate the large blackouts, there can be an increase in frequency of the small ones. The negative effects of some mitigation measures may not necessarily appear right away. They can cause a slowly worsening of the system performance over an extended period of time. That may increase the difficulties in assessing the effectiveness of a measure, and in identifying the cause of worsening of operational conditions.

The importance of developing methods of blackout mitigation can only increase as the power system not only gradually evolves as assumed in this paper, but also changes its character through changes in the nature of generation, loads, and regulation. These additional changes will also be shaped by complex dynamics, but the needs for analysis are greater, because there is less assurance that the historical patterns of high reliability will persist.

Our complex system approach, which implies interdependence between large and small blackouts, should be contrasted with an approach in which large and small blackouts occur $s$-independently as uncorrelated events. The difference between the two approaches cannot be deduced from a frequency distribution of blackout sizes (for these could be the same in both approaches), but from assumptions about the dynamics governing the system that produce these statistics. The complex system approach to risk analysis illustrated here is novel in that it analyses the long-term, steady state risk of failure in a system that is dynamically evolving as the system is upgrading in response to increasing demand. This approach is different than evaluating the risk of failure in a system that is assumed to be fixed. 
We hope that this initial work on risk assessment and mitigation of blackouts of upgrading transmission systems will spur general interest in the risk analysis of complex dynamical infrastructure systems.

\section{REFERENCES}

[1] H. Ren, I. Dobson, and B. A. Carreras, "Long-term effect of the n-1 criterion on cascading line outages in an evolving power transmission grid," IEEE Trans. Power Systems, vol. 23, no. 3, pp. 1217-1225, August 2008 .

[2] The Disturbance Analysis Working Group (DAWG), information on blackouts in North America [Online]. Available: http://www.nerc.com

[3] B. A. Carreras, D. E. Newman, I. Dobson, and A. B. Poole, "Initial evidence for self organized criticality in electric power system blackouts," in Thirty-third Hawaii International Conference on System Sciences, Maui, Hawaii, January 2000.

[4] B. A. Carreras, D. E. Newman, I. Dobson, and A. B. Poole, "Evidence for self-organized criticality in electric power system blackouts," in 34th Hawaii International Conference on System Sciences, Maui, Hawaii, Jan. 2001

[5] J. Chen, J. S. Thorp, and M. Parashar, "Analysis of electric power system disturbance data," in 34th Hawaii International Conference on System Sciences, Maui, Hawaii, Jan. 2001.

[6] B. A. Carreras, D. E. Newman, I. Dobson, and A. B. Poole, "Evidence for self-organized criticality in a time series of electric power system blackouts," IEEE Trans. Circuits and Systems Part I, vol. 51, no. 9, pp. 1733-1740, Sep. 2004.

[7] Å. J. Holmgren and S. Molin, "Using disturbance data to assess vulnerability of electric power delivery systems," Journal of Infrastructure Systems, pp. 243-251, December 2006.

[8] J. Ø. H. Bakke, A. Hansen, and J. Kertész, "Failures and avalanches in complex networks," Europhysics Letters, vol. 76, no. 4, pp. 717-723, 2006.

[9] G. Ancell, C. Edwards, and V. Krichtal, "Is a large scale blackout of the New Zealand power system inevitable?," in Electricity Engineers Association 2005 Conference "Implementing New Zealands Energy Options", Aukland, New Zealand, June 2005.

[10] X. Weng, Y. Hong, A. Xue, and S. Mei, "Failure analysis on China power grid based on power law," Journal of Control Theory and Applications, vol. 4, no. 3, pp. 235-238, August 2006.

[11] Y. Qun and G. Jianbo, "Self-organized criticality and its application in power system collapse prevention," in International Conference on Power System Technology, Chongqing, China, October 2006.

[12] M. E. J. Newman, "Power laws, Pareto distributions and Zipf's law," Contemporary Physics, vol. 46, pp. 323-351, 2005.

[13] B. A. Carreras, V. E. Lynch, I. Dobson, and D. E. Newman, "Complex dynamics of blackouts in power transmission systems," Chaos, vol. 14, no. 3, pp. 643-652, September 2004.

[14] B. A. Carreras, V. E. Lynch, I. Dobson, and D. E. Newman, "Critical points and transitions in a power transmission model," Chaos, vol. 12, no. 4, pp. 985-994, December 2002.

[15] The IEEE 118 node test system [Online]. Available: http://www.ee. washington.edu/research/pstca/

[16] C. Perrow, Normal Accidents. Princeton, NJ: Princeton, 1984.

[17] I. Dobson, D. E. Newman, B. A. Carreras, and V. E. Lynch, "An initial complex systems analysis of the risks of blackouts in power transmission systems," in Power Systems and Communications Infrastructures for the future, Beijing, China, September 2002.

[18] B. A. Carreras, V. E. Lynch, I. Dobson, and D. E. Newman, "Blackout mitigation assessment in power transmission systems," in Thirty-sixth Hawaii International Conference on System Sciences, Hawaii, January 2003.

[19] "Initial review of methods for cascading failure analysis in electric power transmission systems," in IEEE Power and Energy Society General Meeting, Pittsburgh, PA, USA, July 2008, IEEE PES CAMS Task Force on Cascading Failure

[20] R. Billinton and R. N. Allan, Reliability Evaluation of Power Systems, second ed. New York: Plenum Press, 1996, ch. Chapter 13.

[21] R. Billinton, J. Otengadjei, and R. Ghajar, "Comparison of 2 alternate methods to establish an interrupted energy assessment rate," IEEE Trans. Power Systems, vol. 2, no. 3, pp. 751-757, August 1987.

[22] D. W. Caves, J. A. Herriges, and R. J. Windle, Customer demand for service reliability, A synthesis of the outage costs literature Electric Power Research Institute report P-6510, September 1989.

[23] K. K. Kariuki and R. N. Allan, "Evaluation of reliability worth and value of lost load," IEE Proceedings-Generation Transmission and Distribution, vol. 143, no. 2, pp. 171-180, March 1996.
[24] J. L. Corwin and W. T. Miles, Impact assessment of the 1977 New York City blackout U.S. Department of Energy, Washington, DC, 1978.

[25] S. M. Rinaldi, J. P. Peerenboom, and T. K. Kelly, "Identifying, understanding and analyzing critical infrastructure dependencies," IEEE Control Systems Magazine, vol. 21, no. 6, pp. 11-25, December 2001.

[26] North American Electricity Reliability Council Working group forum on critical infrastructure protection, An approach to action for the electricity sector, Appendices E and F June 2001.

[27] CIGRÉ Task Force 38.06.01, Methods to consider customer interruption costs in power system analysis CIGRÉ report 191, August 2001.

[28] S. D. Sagan, "The problem of redundancy problem: why more nuclear security forces may produce less nuclear security," Risk Analysis, vol. 24, no. 4, pp. 935-946, 2004.

[29] P. Bak, C. Tang, and K. Weisenfeld, "Self-organized criticality: An explanation of 1/f noise," Phys. Rev. Lett., vol. 59, pp. 381-384, 1987.

[30] B. Drossel and F. Schwabl, "Self-organized critical forest-fire model," Phys Rev. Lett., vol. 69, p. 1629, 1992.

[31] P. Bak, How Nature Works: The Science of Self-Organized Criticality. New York: Copernicus books, 1996.

[32] H. J. Jensen, Self-Organized Criticality. Cambridge, U.K.: Cambridge University Press, 1998.

[33] I. Dobson, B. A. Carreras, V. E. Lynch, and D. E. Newman, "Complex systems analysis of series of blackouts: Cascading failure, critical points, and self-organization," Chaos, vol. 17, p. 026103, June 2007.

[34] D. P. Nedic, I. Dobson, D. S. Kirschen, B. A. Carreras, and V. E. Lynch, "Criticality in a cascading failure blackout model," International Journal of Electrical Power and Energy Systems, vol. 28, pp. 627-633, 2006.

[35] B. Stott and E. Hobson, "Power system security control calculations using linear programming, Part I and Part II," IEEE Trans. Power Apparatus and Systems, vol. PAS-97, no. 5, pp. 1713-1731, Sept./Oct. 1978.

[36] J. Chen, J. S. Thorp, and I. Dobson, "Cascading dynamics and mitigation assessment in power system disturbances via a hidden failure model," International Journal of Electrical Power and Energy Systems, vol. 27, no. 4, pp. 318-326, May 2005.

David E. Newman received the B.S. degree in physics and mathematics from the University of Pittsburgh, Pittsburgh, PA in 1983 and the Ph.D. degree in physics from the University of Wisconsin, Madison, in 1993. He was a Wigner Fellow and Research Scientist at Oak Ridge National Laboratory, Oak Ridge, TN, from 1993 to 1998. In 1998, he joined the University of Alaska, Fairbanks, where he is now a Professor of Physics.

Benjamin A. Carreras received the Licenciado en Ciencias degree in physics from the University of Barcelona, Spain, in 1965, and the Ph.D. degree in physics from Valencia University, Spain, in 1968. He has been a Researcher and a Professor at the University of Madrid, Spain, Glasgow University, U.K., Daresbury Nuclear Physics Laboratory, Warrington, U.K., Junta de Energia Nuclear, Madrid, Spain, and the Institute for Advanced Study, Princeton, NJ He was a Corporate Fellow at Oak Ridge National Laboratory, Oak Ridge, TN $\mathrm{He}$ is now Principal Scientist at BACV Solutions, Inc. Oak Ridge, TN. Dr. Carreras is a Fellow of the American Physical Society.

Vickie E. Lynch received the BS in Mathematics from Union University in Jackson, Tennessee in 1977 and the MS in Applied Mathematics from the University of Tennessee in 1979. She is a Research Staff member of the Modeling and Simulation Group in the Computational Science and Engineering Division, Oak Ridge National Laboratory. Her research interests include numerical calculations of fusion energy turbulence and transport, numerical methods for solving PDEs with fractional derivatives, analysis of experimental data, tracer particles in 3-D parallel turbulence calculations, pictures of quasi-coherent resistive ballooning structures, and calculations of the dynamics of blackouts in electric power systems. She has received Martin Marietta Energy Systems Technical Achievement Award and Joint Publication Awards and a Technical achievement award from the Association of Women in Science.

Ian Dobson (F'06) received the BA degree in Mathematics from Cambridge, England in 1978 and the $\mathrm{PhD}$ degree in Electrical Engineering from Cornell University USA in 1989. He worked as a systems analyst for the British firm EASAMS Ltd. from 1978 to 1983. In 1989 he joined the University of Wisconsin-Madison USA, where he is now Professor in electrical and computer engineering. His interests are cascading failure risk, complex systems, blackouts, electric power system stability, and nonlinear dynamics. 